\title{
Quantitative assessment of synovitis in patients with rheumatoid arthritis using fluorescence optical imaging
}

Valentin S Schäfer ${ }^{1 *}$, Wolfgang Hartung ${ }^{1}$, Patrick Hoffstetter ${ }^{2}$, Jörn Berger ${ }^{3}$, Christian Stroszczynski ${ }^{4}$, Martina Müller ${ }^{5}$, Martin Fleck ${ }^{1,5}$ and Boris Ehrenstein ${ }^{1}$

\begin{abstract}
Introduction: To prospectively evaluate quantitative assessment of fluorescence optical imaging (FOI) for differentiation of synovitic from non-synovitic joints in patients suffering from rheumatoid arthritis (RA).

Methods: $\mathrm{FOI}$ of the hands was performed in patients with active RA, and a stratified quantitative fluorescence readout (FLRO) of 3 phases (1-120 s; 121-240 s; 241-360 s) was generated for 5 individual joints of the clinical predominant hand (carpal joint, metacarpophalangeal and proximal interphalangeal joints of digits II \& III). To dissect the effect of the overall perfusion of the hand from the perfusion due to synovitis, a fluorescence ratio (FLRA) was additionally calculated, dividing each FLRO by the readout of the eponychium of digit II. The mean FLRO and FLRA were compared between joints with absent vs. present synovitis determined by clinical examination, grayscale, color Doppler ultrasonography, or magnetic resonance imaging (MRI).
\end{abstract}

Results: The analysis for 90 individual joints from 18 patients yielded FLRO ranging from 4.4 to $49.0 \times 10^{3}$, and FLRAs ranging from 0.37 to 2.27 . Overall, the analyses based on the FLRA revealed a higher discrimination than the analyses related to the FLRO, demonstrating most significant differences in phases 2 and 3. A sensitivity of 26/39 (67\%) and a specificity of 31/40 (77\%) were calculated for FLRA of phase 3 using a cut-off value of more than 1.2 to detect MRI-confirmed synovitis with FOI.

Conclusions: FOI has a potential for visualizing synovitis in subjects with RA. For adequate FOI interpretation, quantitative analysis should be based on the novel FLRA calculated for phases 2 and 3.

\section{Introduction}

Rheumatoid arthritis (RA) is an inflammatory disorder involving multiple joints $[1,2]$. It is the most common chronic inflammatory joint disease with a prevalence of 0.5 to $1.0 \%[3,4]$. Persistent synovitis leads to massive joint destruction, and eventually causes irreversible disability in patients. It is of uttermost importance to recognize early synovitis, before substantial joint damage occurs and to start early and aggressive therapy in order to improve short- and long-term outcomes [5-7]. Therefore, sensitive and specific tools for early diagnosis of RA are necessary $[5,6]$. The clinical examination (CE) is a prerequisite, but may miss subclinical inflammation in patients with early

\footnotetext{
* Correspondence: va.schaefer@asklepios.com

'Department of Rheumatology and Clinical Immunology, Asklepios Medical Center Bad Abbach, 93077 Bad Abbach, Germany

Full list of author information is available at the end of the article
}

disease as well as in those who are in clinical remission under treatment [8]. Conventional $\mathrm{x}$-ray examination is often used as an indicator of prognosis and represents the standard outcome measure of disease progression, but does not display the current disease activity [9]. Ultrasonography (US) is a valid tool in the assessment of patients with synovitis and in scoring the clinical activity [10-12]. US is, however, a time-consuming, and operator-dependent technique and might miss signs of early arthritis [13-15]. Magnetic resonance imaging (MRI) has proven to be the strongest independent predictor of radiographic progression in patients with RA [5,9]. MRI, however, is costly, time-consuming, and not ubiquitously available. For fast and dynamic assessment of joint inflammation, US is more widely available than MRI in daily clinical practice [16]. The disadvantage of US is that it is a time-consuming method, and, apart from in clinical studies, the examination 
is usually limited to a reduced number of joints due to time constraints.

Fluorescence optical imaging (FOI) is a new, noninvasive and non-ionizing imaging technology with fast acquisition times $[17,18]$. The major drawback of FOI is the limited tissue penetration of light; however, as inflammatory arthopathies typically affect the small joints of the hands and feet, this is not necessarily a significant limitation for this imaging method [19]. Under various experimental conditions, FOI proved to correspond to synovitis $[17,18,20-24]$. In those experiments, early hyperemia of inflamed joints could be diagnosed by recording scattering and absorption patterns of light transmitted through inflamed finger joints. This approach has already been tested in humans $[21,25]$, which led to the development of an FOI system with fixed optical geometry (Xiralite X4; Mivenion GmbH, Berlin, Germany). The fluorescent dye indocyanine green (ICG) appears to be an appropriate tracer, because it has been shown to enhance inflamed joints $[20,26]$. Furthermore, this substance has been approved by the Federal Drugs Administration. In a study by Scheel et al. [27] FOI provided information about the inflammation status of finger joints with a sensitivity and specificity of $80 \%$ and $89 \%$, respectively. In a clinical study by Werner et al. [8] FOI had, taking MRI as a reference, a sensitivity of $76 \%$ and a specificity of $54 \%$. Recently, another study by Meier et al. [19] reported that FOI had a sensitivity of $39.6 \%$ and a specificity of $85.2 \%$, compared with MRI demonstrating conflicting results regarding the reliability of this new method. In 2011 Dziekan et al. [28] proposed a quantitative analysis of FOI using normalized variances of fluorescence time correlation functions. In their pivotal pilot study, the joints of healthy volunteers and patients with RA were compared, but no other imaging techniques besides FOI were employed. Therefore, the purpose of this study was to establish a novel quantitative readout for ICG-enhanced FOI to allow more accurate joint assessment and to prospectively compare this imaging method with CE, US, and contrast enhanced 1.5 T MRI for detection of inflamed and non-inflamed joints of the hands in patients with proven RA.

\section{Methods}

\section{Patients}

Patients with known RA seeking routine clinical care and reporting symptoms suggestive of wrist and/or finger joint involvement were asked to participate in the study. Inclusion criteria were an established diagnosis of RA according to the American College of Rheumatology criteria from 1987, elevated rheumatoid factor (RF), and/or elevated anti-cyclic citrullinated peptide (anti-CCP) antibodies, and reported symptoms suggestive of wrist and/or finger-joint involvement. Exclusion criteria were pregnancy, renal failure, increased skin pigmentation [29], known allergy against iodine, ICG or gadolinium, or other contraindications for MRI. The study was performed in compliance with the Declaration of Helsinki. The study protocol was approved by the ethics committee of the University Medical Center Regensburg. All study participants signed consent forms after receiving appropriate written and oral information prior to enrollment.

Demographical data (sex, age, disease duration) and laboratory data ( $\mathrm{RF}$, anti-CCP, serum $\mathrm{C}$ reactive protein (CRP) and erythrocyte sedimentation rate (ESR)) were derived by chart review. All patients received a standardized rheumatologic CE of the 28 joints included in the Disease Activity Score 28 (DAS28, [30]) by one of the authors (V.S. or W.H.) using a bimanual technique, documenting tender and swollen status for each joint. The DAS28 was calculated for each patient [30].

All data regarding clinical and imaging studies were obtained within two days after inclusion for each enrolled patient.

\section{Ultrasonography}

The joints included in the US7 score (wrist, metacarpophalangeal (MCP) and proximal interphalangeal (PIP) joints of the index (II) and middle (III) finger of the clinically dominant hand, and the metatarsophalangeal (MTP) joints of the second (II) and fifth (V) toe of the clinical predominant foot) were examined by ultrasonography by one of the authors (V.S. or W.H.) using a LOGIQ E9 (GE, Munich, Germany) ultrasound machine with a linear transducer (ML 6-15) with $15 \mathrm{MHz}$ frequency [31]. Each joint was rated separately semiquantitatively (grades 0 to 3) for distension of the capsule due to synovitis with grey scale ultrasonography (GSUS) and for synovial vascularity with color Doppler ultrasonography (CDUS). The presence of synovitis was concluded for each mode if at least grade 1 findings were observed. For each patient the US7 score was calculated [31].

\section{Magnetic resonance imaging}

For each patient a gadolinium-enhanced MRI of the clinically dominant hand was performed using a $1.5 \mathrm{~T}$ high-field MR scanner. The main focus of the MRI examination was the evaluation of the carpal and MCP joints, and if included in the examination field also the evaluation of the PIP joints. The examination protocol was performed analog to routine clinical practice and in line with the recommendations of the RAMRIS (Rheumatoid Arthritis MRI Scoring system) [32]. Fat saturated proton or T2-weighted and contrast-enhanced T1-weighted sequences were used in at least one coronal and axial plane. Additional unenhanced T1-weighted sequences were acquired in coronal plane. One of the 18 patients was examined with fat saturated proton-weighted and T2-weighted sequences, and the other 
patients were analyzed utilizing only proton-weighted images. For all patients the contrast-enhanced T1-weighted images were fat saturated using spectral saturating technique. Gadoteric acid (Dotarem', Guerbet, Villepinte, France) was applied as intravenous contrast agent using a dosage of $0.2 \mathrm{ml} / \mathrm{kg}$. (The majority of patients were examined using the following MRI protocol: prone position with the hand placed over the head in an extremity surface flex coil, coronal fat saturated proton-weighted turbo spin echo (Cor PD TSE fs), echo time (TE) 29/repetition time (TR) 2260, slice thickness (ST) $2.5 \mathrm{~mm}$, gap $0.25 \mathrm{~mm}$, field of view (FOV) $240 \times 320 \mathrm{~mm}^{2}$, scan time: 1 minute 40 seconds. Sagittal fat saturated proton-weighted turbo spin echo (Sag PD TSE fs), TE 29/TR 3630, ST $3 \mathrm{~mm}$, gap $0.3 \mathrm{~mm}$, FOV $240 \times 320$ $\mathrm{mm}^{2}$, scan time: 1 minute 45 seconds. Coronal T1-weighted turbo spin echo (Cor T1 TSE), TE 14/TR 586, ST $2.5 \mathrm{~mm}$, gap $0.25 \mathrm{~mm}$, FOV $256 \times 320 \mathrm{~mm}^{2}$, scan time: 2 minutes 16 seconds. Axial contrast-enhanced T1-weighted fat saturated turbo spin echo (Axial CE T1 TSE fs), TE 14/TR 732, ST 2.5 $\mathrm{mm}$, gap $0.25 \mathrm{~mm}$, FOV $236 \times 192 \mathrm{~mm}^{2}$, scan time: 1 minute 40 seconds.) All MRI scans were read according to the RAMRIS scoring system by an experienced radiologist (P.H., seven years of MRI experience) blinded to clinical and ultrasonography data. The presence of synovitis was concluded for each joint, if at least grade one findings were observed. Additionally, the extensor tendons overlying the joint were evaluated for the presence of tenosynovitis. The RAMRIS score has been calculated for each patient.

\section{Fluorescence optical imaging}

A commercially available, near-infrared fluorescence imaging system (Xiralite X4, Mivenion GmbH, Berlin, Germany) was used in this study. The working principle is based on the excitation of ICG dye by light emitting diodes and the detection of fluorescence signals with a sensitive camera. The instrument is controlled by a complementary PC and records image sequences with a standardized frame rate of one frame (image) per second over a period of six minutes. Thus, a stack of 360 images is provided for each individual examination.

The FOI examination followed a standardized procedure: both hands were placed on a preformed hand rest. Ten seconds after starting the examination, an ICG bolus ( 0.1 $\mathrm{mg} / \mathrm{kg}$ ) was injected manually in the cubital vein over the period of approximately one second (ICG-Pulsion, Pulsion, Feldkirchen, Germany). Any alteration of fluorophor concentration can be depicted as alteration of signal intensity. Due to hepatic clearance of ICG, FOI signals decay with a time constant of typically three to four minutes. In a pilot study, it was found that the relative FOI signal intensities within one frame do not change significantly after an imaging period of six minutes. Therefore, this duration was determined as the standard examination time.
FOI findings were analyzed using software provided by the manufacturer (Xiralyze (version 1.0.3), Mivenion $\mathrm{GmbH}$, Berlin, Germany). Electronically generated composite images $(\mathrm{CI})$ were calculated from the mean fluorescence signal intensities of an image stack of sequential subsets of acquired images. In line with two previous publications, we defined $\mathrm{CI}$ for three sequential phases with a duration of 120 seconds each ( 1 to 120 seconds, 121 to 240 seconds, and 241 to 360 seconds) $[8,19]$. To allow for quantitative assessment, five joints (wrist, MCP II and III, and PIP II and III) of each patient were analyzed using circular regions of interest (ROI), with the size of each ROI defined according to the anatomical size of the corresponding joint (a diameter of 22 pixel for wrists, 12 pixel for MCP- and 10 pixel for PIP-joints; see Figure 1). The resulting fluorescence readout (FLRO) represents, therefore, the mean fluorescence intensity per pixel of the analyzed CI.

To evaluate the influence of the general perfusion of the whole hand on the readout of individual joints, an additional analysis method was developed: the FLRO at the eponychium of the index finger was determined in each patient, an anatomical site not known to be involved in inflammatory processes in patients with RA (using a circular ROI with a diameter of five pixel, placed centrally over the eponychium). In order to standardize individual joint FOI readout results for the general perfusion, we established a fluorescence ratio (FLRA) for each joint by dividing the readout of the joint ROI by the readout of the eponychium of the index finger.

\section{Statistical analysis}

Data evaluation and statistical analyses were performed using SPSS software (version 20, IBM, Ehningen, Germany). We compared the mean FLRO and mean FLRAs of joints with compared with those without evidence of synovitis derived by clinical evaluation (tender or swollen joints) or by established imaging techniques (GSUS, CDUS, MRI) for all three phases and the sum of the three phases using Student's $t$-test statistic. Statistical significance was concluded when two-sided $P$ values were below 0.05 .

\section{Results}

The study population included 18 subjects, eight women $(44 \%)$ and 10 men (56\%), with a mean ( \pm standard deviation (SD)) age of $63.0( \pm 10.0)$ years. All subjects tolerated the procedure well, and no adverse events were observed. Patient's clinical and laboratory characteristics, and US and MRI scores are displayed in Table 1. To illustrate the comparison of the employed imaging methods, Figure 2 displays the carpal arthritis of a 50-year-old subject with RA.

A total of 90 joints from the 18 patients were analyzed (18 carpal, $36 \mathrm{MCP}$, and 36 PIP joints). All 90 joints were evaluated by CE and US and 79 by MRI. The CE revealed 


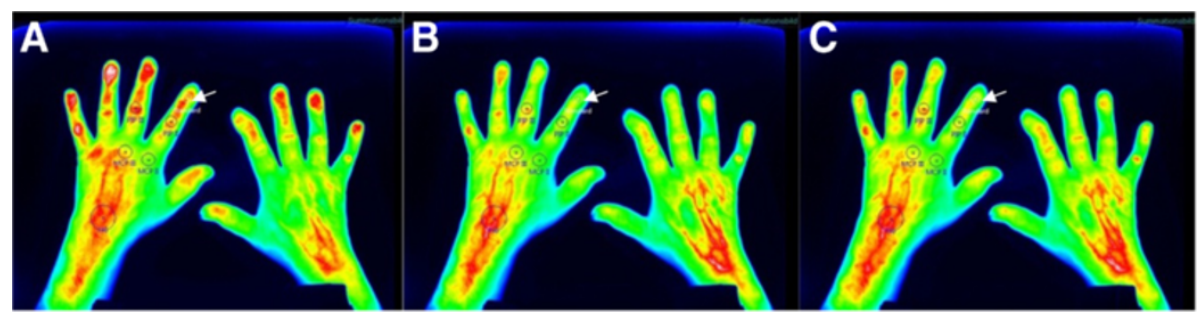

Figure 1 FOI images of a female RA patient. Fluorescence optical imaging composite images of the hands of a female patient with rheumatoid arthritis: (A) phase 1 (1 to 120 seconds); (B) phase 2 (121 to 240 seconds), and (C) phase 3 (241 to 360 seconds). Depicted are the placement of the regions of interest for the carpal, metacarpalphalangeal (MCP), and proximal interphalangeal (PIP) joint of the index (II) and middle (III) fingers and the control region at the eponychium of the index finger (white arrow).

26 of 90 (29\%) swollen joints (SJ) and 44 of $90(49 \%)$ tender joints (TJ). The GSUS revealed 39 of $90(43 \%)$, CDUS revealed 23 of 90 (26\%) and MRI revealed 39 of 79 (49\%) joints that displayed findings consistent with active synovitis. Four of the 79 joints (5\%) evaluated by MRI also displayed tenosynovitis of the overlying extensor tendons, but in all four instances the adjoining joint also showed signs of synovitis.

The quantitative analysis for individual joints of the FLRO yielded values ranging from 4.4 to $49.0 \times 10^{3}$ (mean $19.1 \pm$ SD 9.5) for phase $1,4.2$ to $47.9 \times 10^{3}$ (mean $19.6 \pm$ SD 9.2) for phase 2 , and 2.6 to $40.9 \times 10^{3}$ (mean $12.8 \pm$ SD 6.8) for phase 3 . The results of the quantitative analysis of the FOI data are displayed in Table 2. The comparison yielded significant differences of the mean FLRO between joints with compared to those without evidence of active synovitis determined by US and MRI, but not by CE. Of note, there were significant differences of the mean FLRO between joints with compared to those without synovitis determined by GSUS and MRI for phases 2 and 3, whereas significant differences have been only observed by CDUS in phases 1 and 2.

Table 1 Patient characteristics and results of laboratory and imaging studies ${ }^{*}$

\begin{tabular}{lc}
\hline All patients, $\mathbf{n}$ (\%) & $\mathbf{1 8 ~ ( 1 0 0 \% )}$ \\
\hline Male, $\mathrm{n}(\%)$ & $10(56 \%)$ \\
Female, $\mathrm{n}(\%)$ & $8(44 \%)$ \\
Age & $63.0 \pm 10.0$ years \\
Disease duration & $4.9 \pm 4.4$ years \\
ESR & $25.4 \pm 25.3 \mathrm{~mm} / \mathrm{hour}$ \\
CRP & $17.2 \pm 19.6 \mathrm{mg} / \mathrm{l}$ \\
CE, tender joints & $7.3 \pm 5.0$ \\
CE, swollen joints & $5.4 \pm 5.4$ \\
Disease activity (DAS28) & $4.6 \pm 1.6$ \\
Ultrasonography score (US7) & $13.8 \pm 9.4$ \\
MRI score (RAMRIS) & $14.6 \pm 10.0$ \\
\hline
\end{tabular}

*Except where indicated otherwise, values are the mean \pm SD. CE clinical examination, CRP C-reactive protein, DAS28 disease activity score of 28 joints, $E S R$ erythrocyte sedimentation rate, $M R I$ magnetic resonance imaging.
To dissect the effect of the overall perfusion of the hand from the perfusion due to active synovitis and to improve FOI results, novel FLRA scores were calculated for each individual joint as described above. The FLRO at the eponychium of the index finger ranged from 8.6 to $42.2 \times 10^{3}$ (mean 20.8 \pm SD 9.5) for phase 1, 5.5 to $36.0 \times 10^{3}$ (mean $16.8 \pm$ SD 7.7) for phase 2, and 3.1 to $25.3 \times 10^{3}$ (mean $10.7 \pm$ SD 5.4) for phase 3. The derived values of the FLRAs ranged from 0.37 to 2.27 (mean $0.97 \pm$ SD 0.39) for phase $1,0.60$ to 2.88 (mean $1.20 \pm$ SD 0.39 ) for phase 2 , and 0.62 to 2.87 (mean $1.23 \pm$ SD 0.39 ) for phase 3. The results of the FLRAs are displayed in Table 3. Compared with the analysis of the FLRO, the FLRA results demonstrated improved discrimination between joints with compared with those without evidence of active synovitis resulting in significant differences of the calculated FLRA values compared with all clinical and imaging techniques in phases 2 and 3. Additionally, analyses comparing FOI with MRI stratified by joint type (carpal, MCP, PIP) were performed (data not shown). Due to the fact that 15 of 18 carpal joints displayed synovitis by MRI, no statistical significant differences were found for FLRO and FLRA for this subgroup analysis. The subgroup analyses of MCP and PIP joints revealed similar findings to the analysis of all joints, with better discrimination of FLRA than FLRO in both subgroups.

A receiver-operating characteristic analysis, comparing the FLRAs of phase 1, phase 2, and phase 3 to detect synovitis confirmed by MRI as gold standard revealed the highest area under the curve for phase $3(0.67)$, whereas lower values have been observed for phase $1(0.58)$ and phase 2 (0.65). Using a cut-off value for the FLRA of phase 3 of more than 1.2 to detect MRI-confirmed synovitis with FOI, a sensitivity of 26 of 39 (67\%; 95\% confidence interval (CI), 51 to $79 \%$ ) and a specificity of 31 of 40 (77\%; $95 \%$ CI, 62 to $88 \%$ ) were calculated, with a sensitivity of 21 of 32 (66\%; $95 \%$ CI, 48 to $80 \%$ ) for grade 1, and five of seven (71\%; 95\% CI, 36 to 92\%) for grade 2 and 3 MRI-detected synovitis. A stratified analysis by joint type utilizing the same cut-off for FLRA of phase 3 to detect synovitis confirmed by MRI revealed a sensitivity/specificity of 


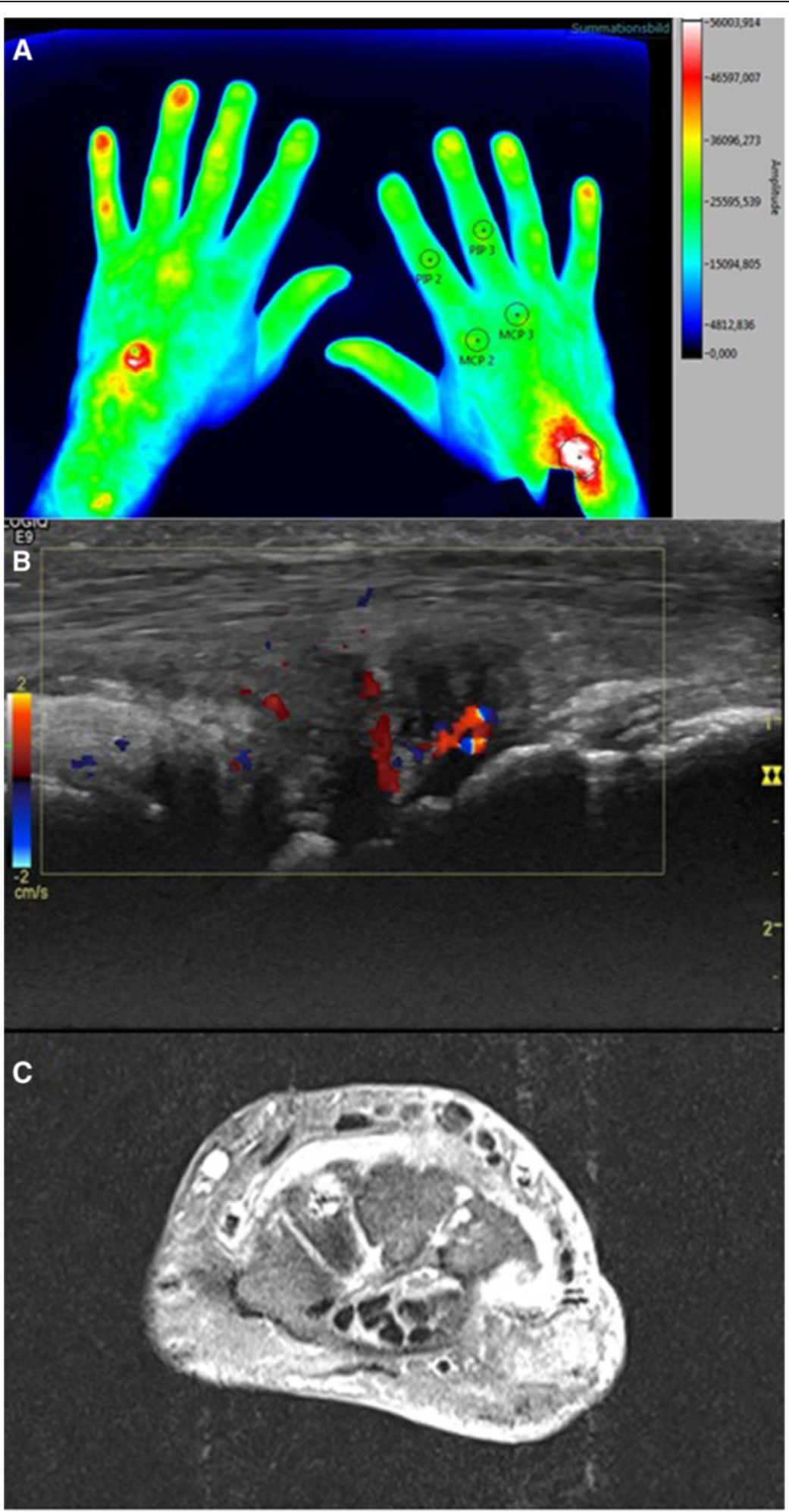

Figure 2 Correlation of FOI with CDUS and MRI. Comparison of the fluorescence optical imaging (FOI) composite image (A) of phase 2 (121 to 240 seconds) with (B) color Doppler ultrasonography (CDUS; showing effusion with hyperperfusion) and (C) magnetic resonance imaging (MRI; contrast-enhanced T1-weighted transverse view displaying grade 2 synovitis) of a 50-year-old male patient with carpal arthritis of the right hand. 
Table 2 Mean fluorescence readout for joints with compared to those without evidence of synovitis determined by clinical examination or established imaging techniques \#

\begin{tabular}{|c|c|c|c|c|c|c|c|}
\hline \multirow[b]{2}{*}{ Synovitis } & \multirow[b]{2}{*}{$n$} & \multicolumn{2}{|c|}{$\begin{array}{c}\text { FOI phase } 1 \\
(1-120 \text { seconds })\end{array}$} & \multicolumn{2}{|c|}{$\begin{array}{c}\text { FOI phase } 2 \\
(121-240 \text { seconds })\end{array}$} & \multicolumn{2}{|c|}{$\begin{array}{c}\text { FOI phase } 3 \\
(241-360 \text { seconds) }\end{array}$} \\
\hline & & mean $\pm S D$ & $P$ & mean $\pm S D$ & $P$ & mean $\pm S D$ & $P$ \\
\hline TJ yes & 44 & $19.1 \pm 11.0$ & ns & $19.6 \pm 9.0$ & $\mathrm{~ns}$ & $12.6 \pm 5.7$ & ns \\
\hline TJ no & 46 & $19.2 \pm 7.8$ & & $19.6 \pm 9.4$ & & $13.0 \pm 7.8$ & \\
\hline SJ yes & 26 & $22.7 \pm 12.6$ & ns & $22.3 \pm 9.8$ & ns & $14.1 \pm 6.2$ & ns \\
\hline SJ no & 64 & $17.7 \pm 7.5$ & & $18.5 \pm 8.7$ & & $12.3 \pm 7.0$ & \\
\hline GSUS yes & 39 & $21.0 \pm 11.4$ & ns & $23.5 \pm 10.4$ & $* *$ & $15.8 \pm 7.9$ & $* *$ \\
\hline GSUS no & 51 & $17.7 \pm 7.5$ & & $16.6 \pm 6.8$ & & $10.5 \pm 4.8$ & \\
\hline CDUS yes & 23 & $24.1 \pm 12.3$ & * & $23.8 \pm 9.3$ & * & $15.0 \pm 5.9$ & ns \\
\hline CDUS no & 67 & $17.5 \pm 7.7$ & & $18.2 \pm 8.7$ & & $12.0 \pm 7.0$ & \\
\hline MRI yes & $39^{\S}$ & $20.9 \pm 10.1$ & ns & $22.3 \pm 9.5$ & $*$ & $14.8 \pm 7.3$ & $*$ \\
\hline MRI no & $40^{\S}$ & $17.8 \pm 9.6$ & & $17.2 \pm 9.2$ & & $10.9 \pm 6.5$ & \\
\hline All & 90 & $19.1 \pm 9.4$ & & $19.6 \pm 9.2$ & & $12.8 \pm 6.8$ & \\
\hline
\end{tabular}

\# Fluorescence readout results are displayed for better readability divided by $10^{3} ;{ }^{\S} 11$ joints could not be evaluated with $\mathrm{MRI} ;{ }^{*} P<0.05 ;{ }^{* *} P<0.01$. CDUS color Doppler ultrasonography, FOI fluorescence optical imaging, GSUS grey-scale ultrasonography, MRI magnetic resonance imaging; ns, comparison was not significant $(P \geq 0.05$ with student's $t$-test), $S D$ standard deviation, $S J$ swollen joint by clinical examination, $T J$ tender joint by clinical examination.

$87 \%$ (95\% CI, 62 to $96 \%) / 0 \%$ (95\% CI, 0 to $56 \%$ ) for carpal joints, $42 \%$ (95\% CI, 23 to $62 \%) / 93 \%$ (95\% CI, 70 to $99 \%$ ) for MCP joints, and 100\% (95\% CI, 57 to 100\%)/77\% (95\% CI, 57 to $90 \%$ ) for PIP joints.

\section{Discussion}

FOI with the Xiralite system is an emerging imaging technology. However, so far only semiquantitative analyses of FOI results have been reported limiting the strength and reliability of this method. Therefore, we aimed to establish a novel scoring system to allow for a quantitative
FOI analysis: introducing and defining circular ROIs for five joints (wrist, MCP II and III, and PIP II and III) FLROs could be calculated representing the mean fluorescence intensity per pixel of the analyzed CI. In order to standardize the quantitative analysis for each patient individually, the FLRO at the eponychium of the index finger has been determined in each patient as a marker of the general perfusion. Dividing the readout of the joint ROI by the readout of the eponychium of the index finger, FLRAs could be calculated for each joint allowing quantitative analysis of FOI results in each patient individually. To validate the

Table 3 Mean fluorescence ratios (standardized for overall perfusion of the hand) for joints with compared with those without evidence of synovitis determined by clinical examination or established imaging techniques \#

\begin{tabular}{|c|c|c|c|c|c|c|c|}
\hline \multirow[b]{2}{*}{ Synovitis } & \multirow[b]{2}{*}{$n$} & \multicolumn{2}{|c|}{$\begin{array}{l}\text { Ratio FOl phase } 1 \\
(1-120 \text { seconds) }\end{array}$} & \multicolumn{2}{|c|}{$\begin{array}{c}\text { Ratio FOI phase } 2 \\
(121-240 \text { seconds) }\end{array}$} & \multicolumn{2}{|c|}{$\begin{array}{l}\text { Ratio FOI phase } 3 \\
(241-360 \text { seconds) }\end{array}$} \\
\hline & & Mean $\pm S D$ & $P$ & Mean $\pm S D$ & $P$ & Mean $\pm S D$ & $P$ \\
\hline TJ yes & 44 & $1.02 \pm 0.45$ & ns & $1.31 \pm 0.48$ & * & $1.33 \pm 0.48$ & * \\
\hline TJ no & 46 & $0.93 \pm 0.33$ & & $1.10 \pm 0.23$ & & $1.13 \pm 0.24$ & \\
\hline SJ yes & 26 & $1.13 \pm 0.50$ & $*$ & $1.43 \pm 0.54$ & $* *$ & $1.45 \pm 0.54$ & $* *$ \\
\hline SJ no & 64 & $0.91 \pm 0.33$ & & $1.11 \pm 0.26$ & & $1.14 \pm 0.26$ & \\
\hline GSUS yes & 39 & $1.06 \pm 0.47$ & $\mathrm{~ns}$ & $1.33 \pm 0.48$ & * & $1.37 \pm 0.48$ & $* *$ \\
\hline GSUS no & 51 & $0.91 \pm 0.32$ & & $1.10 \pm 0.26$ & & $1.12 \pm 0.26$ & \\
\hline CDUS yes & 23 & $1.22 \pm 0.46$ & $* *$ & $1.52 \pm 0.52$ & $* *$ & $1.53 \pm 0.53$ & $* *$ \\
\hline CDUS no & 67 & $0.89 \pm 0.33$ & & $1.09 \pm 0.26$ & & $1.12 \pm 0.26$ & \\
\hline MRI yes & $39^{\S}$ & $1.11 \pm 0.46$ & $* *$ & $1.38 \pm 0.47$ & $* * *$ & $1.41 \pm 0.46$ & $* * *$ \\
\hline MRI no & $40^{\S}$ & $0.86 \pm 0.33$ & & $1.06 \pm 0.26$ & & $1.08 \pm 0.26$ & \\
\hline All & 90 & $0.97 \pm 0.39$ & & $1.20 \pm 0.39$ & & $1.22 \pm 0.39$ & \\
\hline
\end{tabular}

\# Fluorescence ratios were derived by dividing the readout of each joint by the readout of the control area of the eponychium of the index finger; ${ }^{\S} 11$ joints could not be evaluated with MRI; ${ }^{*} P<0.05 ;{ }^{* *} P<0.01 ;{ }^{* * *} P<0.001$.

CDUS, color Doppler ultrasonography; FOI, fluorescence optical imaging; GSUS, grey-scale ultrasonography; MRI, magnetic resonance imaging; $n s$, comparison was not significant $(P \geq 0.05$ with student's $t$-test); SD, standard deviation; $S J$, swollen joint by clinical examination; $T J$, tender joint by clinical examination. 
novel quantitative FOI scores, other imaging techniques (GSUS, CDUS, and MRI) as well as CE were applied to compare differentiation of joints with and joints without evidence of synovitis. To our knowledge this is the first study evaluating the ability of this novel imaging modality comparing quantitative fluorescence readout to synovitis detected by MRI and US.

In arthritic conditions, angiogenesis is highly dysregulated [33]. Furthermore, hypervascularisation and angiogenesis of the synovial membrane are a hallmark in RA patients [34] and strongly linked to bone destruction. The degree of synovial vascularisation correlates well with the disease activity of a given joint [35,36], as well as radiographic progression [37] and with the therapeutic response in patients with RA [38]. Therefore, FOI might be a useful tool to detect and monitor disease activity in RA patients by visualization of microperfusion changes in affected joints.

Using the quantitative approach for the evaluation of the obtained FOI results, significant differences of the mean FLRO have been observed between synovitic and non-synovitic joints for the results of phases 2 and 3 , which were even more striking after standardization by calculating the FLRAs. Unfortunately, no definite cut-off values for perfect separation of synovitic from nonsynovitic joints could be established in our pilot study. However, a sensitivity of $67 \%$ and a specificity of $77 \%$ was calculated using a cut-off value for the FLRAs of phase 3 of more than 1.2 to detect MRI-confirmed synovitis with FOI, which demonstrates the diagnostic yield of this novel imaging method. The subgroup analyses stratified by joint type revealed for this cut-off a higher sensitivity for carpal and PIP joints than for MCP joints. Larger future studies should address if different joint types deserve distinct cut-offs for FLRA interpretation.

In each FOI sequence, three phases were scaled, giving different results in comparison to the other imaging modalities. In the publication by Werner et al. [8] phase 1 displayed the highest agreement with CE and CDUS, whereas the highest sensitivity was observed in phase 2, comparable with the sensitivity of US in relation to MRI. Using MRI as standard of reference, the FOI system displayed a sensitivity of $76 \%$ and a specificity of $54 \%$ using different analysis parameters. Consistent to these findings, a sensitivity of $67 \%$ and a specificity of $77 \%$ were calculated for FOI in our study compared with MRI findings. With regard to the different phases analyzed, our results differ partially to the previous observations, as phase 1 did not show similar significant results, whereas phases 2 and 3 correlated best with all imaging modalities following standardization.

In a recent study by Meier et al. [19], FOI was compared with CE and three T-weighted MRI. Using MRI as standard of reference, FOI displayed a sensitivity of $39.6 \%$ and a specificity of $85.2 \%$. In that study, the diagnostic accuracy of the FOI was altogether lower and particularly limited in mild synovitis, whereas it performed substantially better in severely inflamed joints.

So far, different methods for interpreting the FOI results have been deployed $[8,19,28]$. Werner et al. [8] used a semiquantitative score, where the signal enhancement has been graded in percentages. In contrast, Meier et al. [19] utilized a semiquantitative assessment where synovitis was graded in each joint ranging from 0 to 3 . As outlined by these investigators, a semiquantitative approach revealed only moderate interreader agreement with poor findings for the PIP and DIP joints, respectively [19]. Although we did not test for interreader agreement formally, our quantitative approach using directly computed FLRO and FLRA circumvents these problems of semiquantitative analyses.

In their study, Dziekan et al. used a ROI placed over the corresponding finger nail to standardize the FLRO of each finger joint to the interpersonal changes of overall perfusion [28]. Their quantitative analyses were not stratified into the three phases, as employed in our and other studies $[8,19,28]$. The authors noted that with their proposed method of quantitative FOI analysis a good separation between asymptomatic and inflamed joints (delineated by clinical examination of patients with RA) was not possible. They mainly attributed that finding to the lack of a 'gold standard', because no other imaging methods (e.g. US or MRI) were employed in their study.

Werner et al. utilized the percentage of surface area of the affected joint to arrive at a ordinal scale from 0 to 3 , whereas the publication by Meier et al. used the criteria of the RAMRIS semiquantitative scoring system [32], developed to evaluate synovitis detected by MRI, also for semiquantitative interpretation of the FOI data $[8,19]$. Both approaches are based on changes of fluorescence intensity of the joint of interest compared with the fluorescence in unaffected joints over time, and are therefore not prone to be affected strongly by the background change of increasing and then decreasing concentrations of ICG. This difference of ICG concentrations during the three phases may explain why FLRA displayed a better discrimination between inflamed and non-inflamed joints then the plain FLRO results.

We are aware of some limitations concerning the image interpretation and quantification of pathological changes. Although the FOI procedure itself is standardized, consistent standards for image adjustment and automated interpretation are not yet established. We have chosen to set ROIs over those finger joints, which are typically involved in RA. This allows for better quantitative examination, but each ROI has to be set manually and the radius of the different ROIs needs still to be more accurately defined. 


\section{Conclusions}

Our pilot study demonstrates that a quantitative assessment of the fluorescence results obtained by FOI is feasible, although so far no definite cut-off value could be delineated that perfectly separates inflamed from non-inflamed joints in patients with RA. Based on the better discrimination of FLRA compared with FLRO we postulate to use FLRA in larger studies to further set and evaluate the diagnostic yield of quantitative interpretation of FOI in the clinical care of patients with suspected or established RA.

\section{Abbreviations}

Anti-CCP antibodies: Anti-cyclic citrullinated peptide antibodies; CDUS: Color Doppler ultrasonography; CE: Clinical examination; CRP: C-reactive protein; DAS 28: Disease activity score of 28 joints; ESR: Erythrocyte sedimentation rate; FLRA: Fluorescence ratio; FLRO: Quantitative fluorescence readout; FOl: Fluorescence optical imaging; FOV: Field of view; GSUS: Grey-scale ultrasonography; ICG: Indocyanine green; MCP: Metacarpophalangeal; MRI: Magnetic resonance imaging; MTP: Metatarsophalangeal; PIP: Proximal interphalangeal; RA: Rheumatoid arthritis; RF: Rheumatoid factor; ROI: Regions of interest; SD: Standard deviation; SJ: Swollen joint; ST: Slice thickness; TE: Echo time; TJ: Tender joint; TR: Repetition time; US: Ultrasonography.

\section{Competing interests}

$J B$ is employed by the manufacturer of the system and software used to perform FOI in this study. All other authors declare no conflict of interest.

\section{Authors' contributions}

VS, WH, MF, and BE designed the study. VS and WH carried out the acquisition and quantification of clinical examination, sonography, and FOI data. PH graded the MRI examinations. JB contributed to the utilization of the FOI quantification software. VS and BE performed the statistical analysis. $\mathrm{VS}, \mathrm{WH}, \mathrm{PH}, \mathrm{CS}, \mathrm{MM}, \mathrm{MF}$, and $\mathrm{BE}$ analyzed and interpreted the data and prepared the manuscript. All authors read and approved the final manuscript.

\section{Acknowledgement}

\section{Funding}

The study was funded by internal research funds of the Department of Rheumatology and Clinical Immunology, Asklepios Medical Center Bad Abbach, Bad Abbach, Germany.

\section{Author details}

'Department of Rheumatology and Clinical Immunology, Asklepios Medical Center Bad Abbach, 93077 Bad Abbach, Germany. ㄹepartment of Radiology, Asklepios Medical Center Bad Abbach, Bad Abbach, Germany. ${ }^{3}$ Mivenion $\mathrm{GmbH}$, Berlin, Germany. ${ }^{4}$ Department of Radiology, University Medical Center Regensburg, Regensburg, Germany. ${ }^{5}$ Department of Internal Medicine (I), University Medical Center Regensburg, Regensburg, Germany.

Received: 1 November 2012 Accepted: 20 August 2013 Published: 18 September 2013

\section{References}

1. Scott DL, Coulton BL, Popert AJ: Long term progression of joint damage in rheumatoid arthritis. Ann Rheum Dis 1986, 45:373-378.

2. Scott DL, Smith C, Kingsley G: Joint damage and disability in rheumatoid arthritis: an updated systematic review. Clin Exp Rheumatol 2003, 21:S20-S27.

3. Gabriel SE: The epidemiology of rheumatoid arthritis. Rheum Dis Clin North Am 2001, 27:269-281.

4. Silman AJ, Pearson JE: Epidemiology and genetics of rheumatoid arthritis. Arthritis Res 2002, 4:S265-S272.

5. Wilke WS, Sweeney TJ, Calabrese LH: Early, aggressive therapy for rheumatoid arthritis: concerns, descriptions, and estimate of outcome. Semin Arthritis Rheum 1993, 23:26-41.
6. Quinn MA, Emery P: Window of opportunity in early rheumatoid arthritis: possibility of altering the disease process with early intervention. Clin Exp Rheumatol 2003, 21:S154-S157.

7. Kim JM, Weisman MH: When does rheumatoid arthritis begin and why do we need to know? Arthritis Rheum 2000, 43:473-484.

8. Werner SG, Langer HE, Ohrndorf S, Bahner M, Schott P, Schwenke C, Schirner M, Bastian H, Lind-Albrecht G, Kurtz B, Burmester GR, Backhaus M: Inflammation assessment in patients with arthritis using a novel in vivo fluorescence optical imaging technology. Ann Rheum Dis 2012, 71:504-510.

9. Backhaus M, Kamradt T, Sandrock D, Loreck D, Fritz J, Wolf KJ, Raber H, Hamm B, Burmester GR, Bollow M: Arthritis of the finger joints: a comprehensive approach comparing conventional radiography, scintigraphy, ultrasound, and contrast-enhanced magnetic resonance imaging. Arthritis Rheum 1999, 42:1232-1245.

10. Szkudlarek M, Court-Payen M, Strandberg C, Klarlund M, Klausen T, Ostergaard M: Power Doppler ultrasonography for assessment of synovitis in the metacarpophalangeal joints of patients with rheumatoid arthritis: a comparison with dynamic magnetic resonance imaging. Arthritis Rheum 2001, 44:2018-2023.

11. Backhaus M, Burmester GR, Sandrock D, Loreck D, Hess D, Scholz A, Blind S, Hamm B, Bollow M: Prospective two year follow up study comparing novel and conventional imaging procedures in patients with arthritic finger joints. Ann Rheum Dis 2002, 61:895-904.

12. Klauser A, Frauscher F, Schirmer M, Halpern E, Pallwein L, Herold M, Helweg G, ZurNedden D: The value of contrast-enhanced color Doppler ultrasound in the detection of vascularization of finger joints in patients with rheumatoid arthritis. Arthritis Rheum 2002, 46:647-653.

13. Brown AK: Using ultrasonography to facilitate best practice in diagnosis and management of RA. Nat Rev Rheumatol 2009, 5:698-706.

14. Szkudlarek M, Klarlund M, Narvestad E, Court-Payen M, Strandberg C, Jensen $\mathrm{KE}$, Thomsen HS, Ostergaard M: Ultrasonography of the metacarpophalangeal and proximal interphalangeal joints in rheumatoid arthritis: a comparison with magnetic resonance imaging, conventional radiography and clinical examination. Arthritis Res Ther 2006, 8:R52.

15. Terslev L, Torp-Pedersen S, Savnik A, von der Recke P, Qvistgaard E, Danneskiold-Samsoe B, Bliddal H: Doppler ultrasound and magnetic resonance imaging of synovial inflammation of the hand in rheumatoid arthritis: a comparative study. Arthritis Rheum 2003, 48:2434-2441.

16. Boesen M, Ellegaard K, Boesen L, Cimmino MA, Jensen PS, Terslev L, TorpPedersen S, Danneskiold-Samsoe B, Bliddal H: Ultrasound doppler score correlates with OMERACT RAMRIS bone marrow oedema and Synovitis score in the wrist joint of patients with rheumatoid arthritis. Ultraschall Med 2011, 33:166-172.

17. Chen WT, Mahmood U, Weissleder R, Tung CH: Arthritis imaging using a near-infrared fluorescence folate-targeted probe. Arthritis Res Ther 2005, 7:R310-R317.

18. Wunder A, Tung $\mathrm{CH}$, Muller-Ladner $\mathrm{U}$, Weissleder R, Mahmood U: In vivo imaging of protease activity in arthritis: a novel approach for monitoring treatment response. Arthritis Rheum 2004, 50:2459-2465.

19. Meier R, Thürmel K, Moog P, Noël PB, Ahari C, Sievert M, Dorn F, Waldt S, Schaeffeler C, Golovko D, Haller B, Ganter C, Weckbach S, Woertler K, Rummeny EJ: Detection of synovitis in the hands of patients with rheumatological disorders: Diagnostic performance of optical imaging in comparison to MRI. Arthritis Rheum 2012, 64:2489-2498.

20. Fischer T, Gemeinhardt I, Wagner S, Stieglitz DV, Schnorr J, Hermann KG, Ebert B, Petzelt D, Macdonald R, Licha K, Schirner M, Krenn V, Kamradt T, Taupitz M: Assessment of unspecific near-infrared dyes in laser-induced fluorescence imaging of experimental arthritis. Acad Radiol 2006, 13:4-13.

21. Fischer T, Ebert B, Voigt J, Macdonald R, Schneider U, Thomas A, Hamm B, Hermann KG: Detection of rheumatoid arthritis using non-specific contrast enhanced fluorescence imaging. Acad Radiol 2010, 17:375-381.

22. Hansch A, Frey O, Hilger I, Sauner D, Haas M, Schmidt D, Kurrat C, Gajda M, Malich A, Brauer R, Kaiser WA: Diagnosis of arthritis using near-infrared fluorochrome Cy5.5. Invest Radiol 2004, 39:626-632.

23. Hansch A, Frey O, Sauner D, Hilger I, Haas M, Malich A, Brauer R, Kaiser WA: In vivo imaging of experimental arthritis with near-infrared fluorescence. Arthritis Rheum 2004, 50:961-967.

24. Simon GH, Daldrup-Link HE, Kau J, Metz S, Schlegel J, Piontek G, Saborowski O, Demos S, Duyster J, Pichler BJ: Optical imaging of experimental arthritis using allogeneic leukocytes labeled with a near-infrared fluorescent probe. Eur J Nucl Med Mol Imaging 2006, 33:998-1006. 
25. Ebert B, Berger J, Voigt J, Macdonald R, Fischer T, Hermann K-G, Licha K, Schirner M: Early Detection of Rheumatoid Arthritis in Humans by Fluorescence Imaging. http://www.opticsinfobase.org/abstract.cfm?URI=BIOMED-2008-BTuF19.

26. Meier R, Krug C, Golovko D, Boddington S, Piontek G, Rudelius M, Sutton EJ, Baur-Melnyk A, Jones EF, Daldrup-Link HE: Indocyanine green-enhanced imaging of antigen-induced arthritis with an integrated optical imaging/ radiography system. Arthritis Rheum 2010, 62:2322-2327.

27. Scheel AK, Krause A, Rheinbaben IM, Metzger G, Rost H, Tresp V, Mayer P, Reuss-Borst M, Muller GA: Assessment of proximal finger joint inflammation in patients with rheumatoid arthritis, using a novel laser-based imaging technique. Arthritis Rheum 2002, 46:1177-1184.

28. Dziekan T, Weissbach C, Voigt J, Ebert B, Macdonald R, Bahner ML, Mahler M, Schirner M, Berliner M, Berliner B, Osel J, Osel I: Detection of rheumatoid arthritis by evaluation of normalized variances of fluorescence time correlation functions. J Biomed Opt 2011, 16:076015.

29. Schäfer VS, Hartung W, Ehrenstein B, Hoffstetter P, Müller M, Fleck M: A novel arthritis in vivo fluorescence optical imaging technology pushed to the limits. Rheumatology 2013, 52:1144-1145.

30. Prevoo ML, van't Hof MA, Kuper HH, van Leeuwen MA, van de Putte $L B$, van Riel PL: Modified disease activity scores that include twenty-eight-joint counts. Development and validation in a prospective longitudinal study of patients with rheumatoid arthritis. Arthritis Rheum 1995, 38:44-48.

31. Backhaus M, Ohrndorf S, Kellner H, Strunk J, Backhaus TM, Hartung W, Sattler H, Albrecht K, Kaufmann J, Becker K, Sörensen H, Meier L, Burmester GR, Schmidt WA: Evaluation of a novel 7-joint ultrasound score in daily rheumatologic practice: a pilot project. Arthritis Rheum 2009, 61:1194-1201.

32. Østergaard M, Peterfy C, Conaghan P, McQueen F, Bird P, Ejbjerg B, Shnier R, O'Connor P, Klarlund M, Emery P, Genant H, Lassere M, Edmonds J: OMERACT Rheumatoid Arthritis Magnetic Resonance Imaging Studies. Core set of MRI acquisitions, joint pathology definitions, and the OMERACT RA-MRI scoring system. J Rheumatol 2003, 30:1385-1386.

33. Kennedy A, Ng CT, Biniecka M, Saber T, Taylor C, O'Sullivan J, Veale DJ, Fearon U: Angiogenesis and blood vessel stability in inflammatory arthritis. Arthritis Rheum 2010, 62:711-721.

34. Pap T, Distler O: Linking angiogenesis to bone destruction in arthritis. Arthritis Rheum 2005, 52:1346-1348.

35. Lindblad S, Hedfors E: Intraarticular variation in synovitis. Local macroscopic and microscopic signs of inflammatory activity are significantly correlated. Arthritis Rheum 1985, 28:977-986.

36. Ostergaard M, Hansen M, Stoltenberg M, Gideon P, Klarlund M, Jensen KE, Lorenzen I: Magnetic resonance imaging-determined synovial membrane volume as a marker of disease activity and a predictor of progressive joint destruction in the wrists of patients with rheumatoid arthritis. Arthritis Rheum 1999, 42:918-929.

37. Fukae J, Kon Y, Henmi M, Sakamoto F, Narita A, Shimizu M, Tanimura K, Matsuhashi M, Kamishima T, Atsumi T, Koike T: Change of synovial vascularity in a single finger joint assessed by power doppler sonography correlated with radiographic change in rheumatoid arthritis: comparative study of a novel quantitative score with a semiquantitative score. Arthritis Care Res (Hoboken) 2010, 62:657-663.

38. Taylor PC, Steuer A, Gruber J, Cosgrove DO, Blomley MJ, Marsters PA, Wagner CL, McClinton C, Maini RN: Comparison of ultrasonographic assessment of synovitis and joint vascularity with radiographic evaluation in a randomized, placebo-controlled study of infliximab therapy in early rheumatoid arthritis. Arthritis Rheum 2004, 50:1107-1116.

Cite this article as: Schäfer et al:: Quantitative assessment of synovitis in patients with rheumatoid arthritis using fluorescence optical imaging. Arthritis Research \& Therapy 2013 15:R124.

\section{Submit your next manuscript to BioMed Central and take full advantage of:}

- Convenient online submission

- Thorough peer review

- No space constraints or color figure charges

- Immediate publication on acceptance

- Inclusion in PubMed, CAS, Scopus and Google Scholar

- Research which is freely available for redistribution

Submit your manuscript at www.biomedcentral.com/submit
C Biomed Central 